\title{
Aproximación al concepto de planificación estratégica agropecuaria
}

\author{
Peña, María Elena* \\ Urdaneta, Fátima** \\ Casanova, Ángel ${ }^{* *}$
}

\section{Resumen}

La empresa ganadera como unidad económica de toma de decisiones en la producción animal, requiere de una planificación acorde con las características del sector, las cuales distan en su concepción de otros autores de la economía. Basado en ello se planteo una investigación con el objetivo de formular una aproximación al concepto de planificación estratégica agropecuaria a partir del análisis de la evidencia empírica contrastada con el referencial teórico de planificación estratégica propuesta por Donnelly et al. (1994). Se seleccionaron fincas registradas en el Programa de Investigación de Sistemas Agropecuarios (PISA) de la Universidad del Zulia, por muestreo aleatorio estratificado con afijación proporcional y la información recolectada fue tratada estadísticamente con distribución de frecuencias. Los resultados muestran un $38,30 \%$ de productores que señalan como atributo de visión empresarial, el ser considerados individuos con respetabilidad entre productores de la zona. Como misión empresarial declaran la tradición familiar. Los objetivos corporativos se diseñan en función de productividad física (42.55\%). El énfasis esta en el manejo animal, no así en el manejo del talento humano e infraestructura y equipos. La planificación estratégica llevada a cabo por lo productores dista del referencial teórico que existe, sin que por ello se pueda asumir que no existen criterios gerenciales aplicados a estos sistemas productivos. La planificación estratégica agropecuaria es un proceso complejo de toma de decisiones llevada a cabo de manera dinámica y flexible que considera la racionalidad económica como principal componente en el diseño de objetivos y planes operativos, y otorga atención a situaciones inmediatas más que aquellas a largo plazo, por lo que se convierte principalmente en una planificación de carácter táctico más que estratégica.

Palabras clave: Gerencia agropecuaria, planificación estratégica, sistemas ganaderos.

Recibido: 10-02-09. Aceptado: 10-06-10

* Docente-Investigadora de la Facultad de Veterinaria de la Universidad del Zulia. marielenapena2006@hotmail.com.

** Docentes-investigadores de la Facultad de Agronomía de la Universidad del Zulia. fatimaurdaneta@cantv.net. 


\section{Approach to the Strategic Planning Concept for Agriculture and Livestock Production}

\section{Abstracts}

The cattle-raising industry, as an economic unit for decision-making in animal production, requires planning according to sector characteristics, which differ in their concept from other economic authors. Based on this viewpoint, a study is proposed to formulate an approach to the concept of strategic livestock production planning, based on analysis of empirical evidence contrasted with the theoretical referent for strategic planning proposed by Donnelly et al. (1994). Farms registered in the Programa de Investigación de Sistemas Agropecuarios (PISA) (Research Program for Agriculture and Livestock Systems) at the University of Zulia were selected, using a stratified random sample with proportional fixation; the information collected was treated statistically with frequency distribution. Results show that $38.30 \%$ of the producers indicate that being considered as respectable individuals among producers in the zone is an attribute of business vision. Family tradition is declared as a business mission. Corporative objectives are designed as a function of physical productivity (42.55\%). Emphasis is on animal management, not on managing human talent, infrastructure and equipment. The strategic planning carried out by the producers differs from the existing theoretical referents; however, one cannot assume that no managerial criteria are applied to these productive systems. Strategic planning for agricultural and livestock production is a complex process of decision making carried out in a dynamic and flexible manner, which considers the economic rationale as its principle component for designing objectives and operative plans and gives attention to immediate more than longterm situations; due to this, it becomes, principally, planning of a tactical more than strategic nature.

Key words: Gerencia agropecuaria, planificación estratégica, sistemas ganaderos.

\section{Introducción}

Los sistemas de producción ganadera constituyen un conjunto de actividades variadas y de complejas relaciones, las cuales son planificadas, diseñadas, ordenadas, coordinadas, dirigidas y/o ejecutadas por el hombre, en su papel de productor-gerente-dueño. Dado que el hombre es ese factor impulsador y organizador de todos los recursos que se manejan en la empresa agropecuaria, posee también la responsabilidad de participar directamente como uno de los recursos productivos de estos sistemas, a la vez de agente decisor del uso y distribución de los mismos.
Según González (1992), estos sistemas ganaderos constituye un sistema alternativo que resulta viable para producir leche y carne, ya que aprovecha para ello los recursos forrajeros disponibles, la tecnología desarrollada a lo largo de los años y la diversidad de condiciones agroclimáticas. Sin embargo, el manejo gerencial llevado a cabo en estos sistemas productivos, ha sido blanco de críticas y en algunos casos de dudas de su existencia, pues surge la interrogante, si realmente se aplican criterios y conceptos gerenciales en el manejo de estas empresas de alta sensibilidad no solo ambiental, sino también económica y de carácter político y social. 
La empresa ganadera es una unidad económica de producción que combina factores y servicios productivos especializados en llevar a cabo un proceso, a fin de producir bienes que destina al mercado (García y Acero de La Cruz, 2008).

Tradicionalmente se ha pensado que manejar una finca, es un asunto serio, pero fácil. Los cambios globales sufridos por estos sistemas de producción, de comprobada complejidad, obligan a los productores agropecuarios a entender la necesidad de asumir el manejo de su finca como una empresa donde objetivos económicos, sociales y ambientales se conjugen de manera efectiva y armónica en búsqueda constante de rentabilidad integral.

Los potreros de nuestras fincas agropecuarias, constituyen el núcleo productivo del negocio ganadero, sin embargo, no son el negocio en sí. Muchas de las decisiones tomadas y aplicadas en el pasado se tomaron sin los beneficios de la planificación, sin embargo, los gerentes modernos reconocen, cada vez más, que la sabiduría y la intuición por si sola no son suficientes para guiar el destino de la organización en el mundo cambiante a que hoy día se somete la empresa (Donnelly et al., 1994). Por lo que se hace necesario asumir un proceso que incluye, no solo el examen de las condiciones externas e internas de la unidad de producción, sino que requiere del diseño de un plan estratégico que defina la naturaleza y sentido de ser de la empresa, a través de la visión, misión de la empresa, y los objetivos que se pretende alcanzar como organización y los planes contentivos de acciones definidas que permitan alcanzar los men- cionados objetivos y cumplir con la misión y visión empresarial establecida.

La mayoría de los gerentes, y en ellos se incluyen a los productores que manejan este tipo de negocio, no desarrollan el plan estratégico de la organización, pues generalmente están más interesados en los beneficios y resultados obtenidos del proceso productivo, en un hoy que no considera mayormente el mañana empresarial (Donnelly et al., 1994). Esto quiere decir que en las organizaciones bien dirigidas existe una relación directa entre la planificación estratégica y los planes de los demás miembros de la empresa encargados de diversas funciones y con la responsabilidad sobre recursos específicos, tal como se observa en el Diagrama 1, donde el plan estratégico contempla la definición de la visión, misión, objetivos y las estrategias. A partir de de ese plan estratégico se generan los planes operativos de la empresa, que según la adaptación realizada de Donnelly et al. (1994:173) corresponden a los planes de manejo, los planes de recurso humano y el plan administrativo.

Continua el autor afirmando que el planeamiento estratégico ayuda a cerrar la brecha entre donde estamos en el presente, y donde queremos estar en el futuro, por lo tanto este planeamiento provee en primer lugar de dirección, a través del establecimiento de metas futuras y en segundo lugar, la lógica (el porqué) de las actividades, es decir, el qué, por quién, cuándo y con qué recursos de la organización.

Todo negocio cuenta con una planificación, aun cuando el proceso formal varíe enormemente dependiendo si la organización es una empresa especializa- 


\section{Diagrama 1 \\ Relación entre el plan estratégico de la organización y los planes operativos}

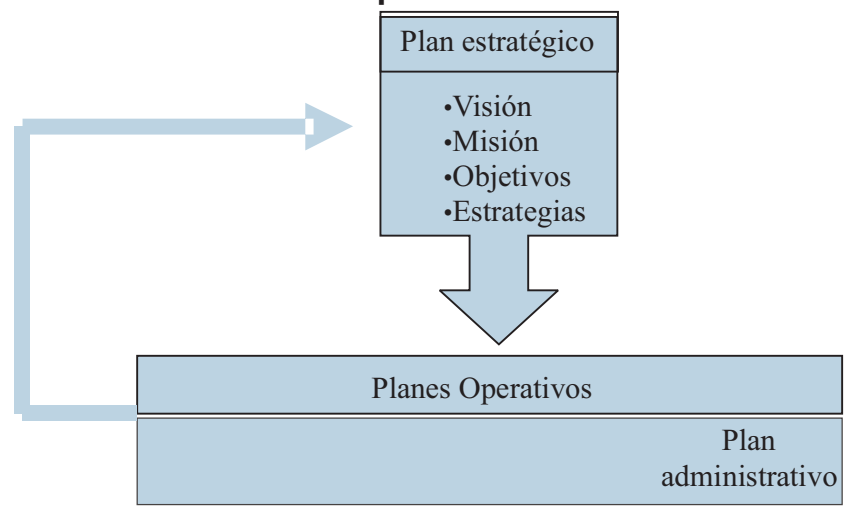

Fuente: Elaboración propia adaptada de lo presentado por Donnelly et al. (1994).

da o diversificada (Hill y Jones, 2001). El proceso es sencillo: los gerentes, de todos los niveles deben ponerse de acuerdo en cuanto a un plan de acción detallado e integrado para ser desarrollado en el período productivo siguiente; este sistema de planificación representa un proceso ordenado y gradual, donde cada paso esta ligado estrechamente al paso anterior.

En la gestión empresarial actual no se concibe una organización que no haya declarado su misión. Desde PYMEs (Pequeñas y Medianas Empresas) hasta EMN (Empresas Multinacionales), incluyendo iglesias, gobiernos locales, grupos ambientalistas y muchos más (Olivera, 2009). Cientos de miles de organizaciones en el mundo entero enfocan (o dicen enfocar) sus esfuerzos al cumplimiento de una misión.

Morillo y Urdaneta (1998) en su definición de doble propósito mencionan como debilidades de este sistema de producción, la falta de evaluación a nivel de campo de los aspectos genéticos, tecno- lógicos, económicos y estadísticos y de investigación básica en fisiología y genética animal sobre poblaciones de ganado mestizo; así como también los problemas gerenciales relacionados con faltas de planificación y control de los sistemas por parte de los propietarios-gerentes, por no contar con los registros apropiados, ni con los recursos humanos calificados, lo cual provoca poca disposición al cambio y a asumir, como lo referido a la aplicación de nuevas tecnologías disponibles entre otros aspectos.

El análisis y diagnóstico del funcionamiento de una empresa, constituye la base donde se apoya el proceso de gerencia agropecuaria. El diagnóstico de una empresa debe conducir a interpretar su funcionamiento, tratando de realizar una interpretación global de la realidad. Es por ello que se realizo una investigación de carácter analítico que implica la reinterpretación de lo analizado en función de algunos criterios, dependiendo de los objetivos del análisis (Hurtado, 2002). Este 
tipo de investigación intenta identificar la sinergia menos evidente de los eventos analizados. En este caso se manifiesta como una contrastación de un evento con otro, o la medida en que un evento contiene o se ajusta a ciertos criterios.

La investigación llevada a cabo es de tipo analítica que atraviesa un estadio descriptivo (Hurtado, 1998; Hernández et al., 1991) y comprendió una fase de recolección de información en el campo, la cual se realizó por medio de una serie de visitas, en cuya oportunidad se utilizó el cuestionario como instrumento para recabar información del período 20052006, además de la revisión de registros y la entrevista e inspecciones a las unidades de producción seleccionadas por el equipo de investigación. Esa previa descripción de los elementos gerenciales presentes en estos sistemas es lo que permite contrastar con el referencial teórico que existe sobre gerencia, de manera que se pueda formular una aproximación a un concepto que abarque la realidad de estas empresas agropecuarias.

Es por ello que se hace necesario realizar investigaciones que arrojen conocimiento acerca de las verdades dimensiones de la planificación estratégica en estas empresas, de allí que se plantea la siguiente investigación con el objetivo general de formular una aproximación al concepto de planificación estratégica agropecuaria a partir del análisis de la evidencia empírica contrastada con el referencial teórico de planificación estratégica propuesta por Donnelly et al. (1994). La investigación llevada a cabo es de tipo analítica que atraviesa un estadio descriptivo (Hurtado, 1998; Hernández et al. 1991) y comprendió una fase de recolec- ción de información en el campo, la cual se realizó por medio de una serie de visitas, en cuya oportunidad se utilizó el cuestionario como instrumento para recabar información del período 20052006 , además de la revisión de registros y la entrevista e inspecciones a las unidades de producción seleccionadas por el equipo de investigación.

El municipio Rosario de Perijá posee 510 fincas dedicadas a la ganadería bovina de doble propósito. Donde el $91.5 \%$ de los sistemas se dedican a producir y vender leche y mautes (animales bovinos de 190 kilogramos de peso) y el restante $8.5 \%$ se dedica a producir y vender leche y novillos (animales bovinos de 350 kilogramos de peso vivo en promedio), basado primordialmente en las características de sus zonas agroecológicas y el desarrollo de un rebaño adaptado a dichas condiciones (www.gdp.infoagro.info.ve, 2008).

La población correspondió a fincas del municipio Rosario de Perijá, estado Zulia registradas en el Programa de Investigación de Sistemas Agropecuarios (PISA) de la Universidad del Zulia, que cumplieron las siguientes características: fincas de doble propósito económicamente activas, con una superficie menor a 2000 ha, propietario con personalidad jurídica o natural, y alta confiabilidad de la información suministrada, en virtud de ser fincas monitoreadas por el equipo de investigación desde 1994.

La muestra estuvo constituida por 47 fincas seleccionadas al azar a través de un muestreo aleatorio estratificado, donde los estratos fueron obtenidos utilizando el criterio de unidades animales, el cual corresponde a la medida que permi- 
te homogeneizar el rebaño que posea la unidad de producción, partiendo del hecho de que una unidad animal representa un animal de $450 \mathrm{~kg}$ de peso, por lo que se genero 3 grupos: menores de $50 \mathrm{UA}$, entre 50 y 300 UA y mayores de 300 UA.

Esa información captada en las unidades de producción permitió evaluar el comportamiento de las mismas en términos de sus aspectos sociales, gerenciales, económicos y técnicos por medio de una serie de indicadores.

La información recogida fue sometida a una codificación que, posteriormente, es vaciada en una matriz de datos construida para cada variable. Éstas, agrupadas en una data general, fueron analizadas con ayuda del Stadistical Analysis System (SAS) Ver. 6.04.

Para cumplir con los objetivos planteados, las variables cuantitativas - continuas fueron analizadas utilizando medidas de tendencia central y de dispersión, específicamente medias y desviaciones estándar. Las variables categóricas se analizaron utilizando como medida de tendencia central la moda. De allí que se utilizó la distribución de frecuencias como técnica estadística para la organización y análisis de la información recolectada, que permitió establecer la discusión de resultados en base a la muestra total sin considerar la estratificación que será utilizada en análisis posteriores a este trabajo.

\section{La planificación estratégica en las fincas de ganadería bovina}

El Plan Estratégico, incluye inevitablemente la construcción en primer lugar de la visión de empresa que responde "a la manera de pensar de todos los miembros de la empresa, sobre todo de la alta dirección" (Hernández, 2006: 312), que se desea del negocio. La visión empresarial es una declaración amplia y suficiente de lo que una empresa es y quiere ser en el futuro, por lo que señala el rumbo, da dirección, es la cadena que une en las empresas el presente con el futuro, sirve de guía en la formulación de las estrategias, a la vez que le proporciona un propósito a la organización. Por ser una proyección de deseo futuro, debe ser suficientemente comprometedora y motivante, de tal manera, que estimule y promueva la pertenencia de todos los miembros de la organización, requiere de una gerencia comprometida, que no le tema al cambio y motive la participación de los integrantes de la compañía en el logro de los objetivos y metas. Debe ser una gerencia que tienda más a la coordinación y al control de gestión que a la operación.

De acuerdo a Serna (1997:177178) para formular la visión de la empresa debe realizarse un proceso de análisis y reflexión que contesten las siguientes interrogantes:

- Si todo tuviera éxito, cómo sería su empresa dentro de 3 años?

- Qué logros de su empresa le gustaría recordar dentro de 5 años?

- Qué innovaciones podrían hacerse a los productos o servicios que ofrece su empresa?

- Qué avances tecnológicos podrían incorporarse?

- Qué otras necesidades y expectativas del cliente podrían satisfacer los productos o servicios que ofrece la empresa, dentro de 3 a 5 años? 
- Qué talentos humanos especializados necesitaría la empresa dentro de 3 a 5 años

Los resultados de la investigación realizada en las fincas manejadas por los productores ganaderos del municipio La Villa del Rosario de Perijá, reflejan algunos atributos sobre los cuales han llegado a formular la visión de su negocio (Tabla 1).

Las respuestas sugeridas por el $38,30 \%$ de los productores señalan una clara tendencia a manifestar como atributo de la visión para el negocio, el ser considerados individuos con un nivel de respetabilidad entre otros productores de la zona, también se visualizan como empresas que posean mayor extensión de tierra que la que actualmente poseen; seguido por aquellos productores que señalan la alta productividad en el aspecto económico, de producción y tecnología $(4,26 \%)$. Estos resultados en algunos casos, responden a algunos de los elementos y las interrogantes planteadas por Serna (1997) para el momento en el cual una organización deba diseñar su visión corporativa.
Sólo el 1\% centró su visión del negocio en la incorporación de paquetes tecnológicos; es de hacer notar, que el presente análisis se refiere a los productores que expresaron alguna respuesta en relación con la visión empresarial, que si bien se observa parcializada hacia alguno de los aspectos del negocio, sin considerar la integralidad de la visión gerencial del mismo, sólo representan el $44,68 \%$ de los que encuestados.

La ausencia de visión empresarial es una característica que define una forma muy sui generis de gerenciar estas empresas agropecuarias, de espaldas a la teoría empresarial moderna, muy común en los sistemas de producción ganaderos, expresado en el $55,32 \%$ de los productores que no respondió a la definición de la visión para su empresa. En oportunidades, este comportamiento esta más relacionado con la necesidad de atender la inmediatez de actividades cotidianas dentro de la finca, que la atención que requiere el proceso de análisis y reflexión en la formulación de lo que se aspira alcanzar como entidad productiva

Tabla 1

\section{Distribución de los atributos de la visión del negocio doble propósito según la opinión del propietario gerente}

\begin{tabular}{lcc}
\hline \multicolumn{1}{c}{ Categoría } & No de fincas & $\%$ \\
\hline $\begin{array}{l}\text { Alta productividad, rentalibilidad, producción de leche, aumentar } \\
\text { unidad animal }\end{array}$ & 2 & 4,26 \\
$\begin{array}{l}\text { Incorporación paquetes tecnológicos } \\
\text { Reconocidos y respetados en la zona como exitosos, productores con } \\
\text { mayor extensión de tierra, considerados como organización modelo de } \\
\text { producción }\end{array}$ & 18 & 38,30 \\
No respondió & 26 & 55,32 \\
Total & 47 & 100 \\
\hline
\end{tabular}

Fuente: elaboración propia, 2006. 
a más largo plazo. Esta actitud se ha constituido en una forma de hacer las cosas que presenta a nuestras unidades productivas como maquinas extractoras y transformadoras de recursos, y no como organizaciones que alcancen objetivos más complejos relacionados con la equidad social, el ambiente y en muchas oportunidades con la productividad. Para que la visión sea eficaz debe ir acompañada de un plan estratégico de mejoramiento y de un plan operativo (Mariño, 2003:18).

La Misión Corporativa... Señala cuál es el negocio de nuestra empresa. Un negocio es una organización que proporciona bienes y servicios con el fin de obtener utilidades (Griffin y Ebert, 1997:4).

Partiendo de esa definición anterior todas las empresas tienen básicamente las mismas razones para justificar su existencia: sobrevivir, crecer, proveer un servicio o producto, generar utilidades, sin embargo, desde el punto de vista organizativo, es necesario que una empresa proponga o asuma razones básicas que las diferencien de sus homologas en el mercado en el cual participan. Por lo tanto, es la Misión la declaración duradera del propósito de la organización realizada por la alta gerencia de esa organización (Serna, 1997:185), en la cual se establece para que existe la misma (Mariño, 2003), cuál es el propósito fundamental, su razón de ser (David,1997), puesto que visualiza la organización a largo plazo, en términos de lo que quiere ser y a quién quiere servir, lo que proporciona dirección y significado a todos los miembros de la organización sin importar el nivel al que pertenecen dentro de la empresa, por lo que proporciona el contexto para formular las líneas específicas de negocios en las cuales se involucrara la empresa y las estrategias mediante las cuales operara; establece el campo en el cual competirá y determina la manera como asignará los recursos y cuál será el modelo general de crecimiento y dirección para el futuro (Goodstein et al., 2004).

Definir la Misión o propósito de la organización es responsabilidad de la alta gerencia empresarial, ya que permite establecer la guía de las decisiones internas y por tanto, debe servir de punto de partida para un mercadeo interno a la vez de apoyarla venta externa.

Para el caso del Municipio Rosario de Perijá, el $91.49 \%$ de los productores manifestaron como razón esencial o propósito primordial de su empresa la tradición familiar que han desarrollado al largo de varias generaciones dedicadas a esta actividad productiva, seguido por $8.51 \%$ de productores que consideran su misión empresarial como una empresa cuyo propósito fundamental es constituirse en principal fuente de ingreso para el núcleo familiar. Este primer valor reseñado pudiera encontrar relación con investigaciones realizadas por Peña et al. (1999) quienes señalan, que aproximadamente, un $46.7 \%$ de los productores de la zona comprendida por los municipios Rosario y Machiques de Perijá han permanecido en la actividad agropecuaria entre 11 a 27 años, presentando una edad promedio de 54 años $(20 \%)$, por lo que la consideración de la tradición familiar como mayor frecuencia de respuesta encuentra sustento en estos aspectos indicados (Tabla 2).

Si bien es cierto que las respuestas obtenidas no reflejan el manejo por parte 


\section{Tabla 2}

\section{Distribución de los criterios de la misión del negocio de doble propósito o la razón por la que existe la empresa según la opinión del productor-gerente de la finca}

\begin{tabular}{ccc}
\hline Categoría & $N^{\circ}$ de fincas & $\%$ \\
\hline Fuente de ingreso & 4 & 8.51 \\
Tradición familiar & 43 & 91.49 \\
\hline
\end{tabular}

Fuente: elaboración propia, 2006.

de los encargados de gerenciar estas unidades de producción, de los aspectos esenciales que deben considerarse al momento de formular la misión de cualquier empresa, se hace necesario que todo productor agropecuario empiece a preguntarse, qué es lo que se espera de él y cual es la función que debe asumir a los fines de transformar su finca en un negocio ganadero; así como definir tres elementos básicos necesarios para establecer la misión: primero, cuál es la necesidad que satisfaces con tu producto o servicio?, segundo, a qué mercado o grupo de consumidores está dirigido tu producto o servicio? Y tercero, de qué manera o a través de qué productos o servicios satisface tu negocio esa necesidad en ese mercado? (Nacional financiera. Banca de Desarrollo, 2008, www.nafin.com). Debe entender que ese propósito no depende solo de él, por lo que se necesita investigar, con los actores comprometidos, cuales son los aspectos o factores que permiten convertir una "buena" finca en una empresa agropecuaria.

Es preciso identificar los elementos que permiten diseñar la misión de la empresa (David, 1997: 83-84). Esta declaración de la propia misión empresarial puede variar, en cuanto a extensión, contenido, formato y especificidad, sin embargo es posible considerar dentro de su formulación los siguientes elementos que pudieran establecerse en su contenido como son:

- Clientes: responde a la interrogante de quiénes son aquellos individuos a quienes es indispensable satisfacer en sus necesidades y expectativas

- Productos o servicios que esos clientes recibirán de la empresa y los cuales deben expresarse claramente en la misión

- Mercados presentes y futuros de la empresa, en este caso es necesario identificar los segmentos o grupos de clientes sobre los cuales la empresa desea tener influencia

- Tecnología y su efecto o importancia considerada dentro de la organización que se maneja

- Interés por la supervivencia, crecimiento y rentabilidad, esta última es la condición de sobrevivencia, puesto que una empresa rentable puede crecer, mantenerse en el mercado, generar utilidades y retribución a sus accionistas, pero ello va a depender no solo del esfuerzo que internamente se realice para alcanzar la eficiencia y eficacia de los recursos utilizados en función de la inversión realizada, sino también del estudio y evaluación del 
entorno cambiante, pero lleno de oportunidades que algunas veces no se presentan de manera tan evidente.

- Principios organizacionales comprendidos por las creencias, valores, aspiraciones que deben ser comunicados a los integrantes de la organización e incorporados en el quehacer diario de la empresa y hacerlos parte de la definición del negocio.

- Compromiso con los grupos de referencia contraídos como responsabilidad ante los accionistas, colaboradores, clientes, la competencia, el medio ambiente y la sociedad en general.

Es por ello, que se hace necesario definir la razón de ser de la empresa desde el punto de vista del negocio sustentable sin olvidar los criterios de responsabilidad social, definir también cuáles serían nuestros obstáculos a corto plazo y cómo se vencerían.

Al observar los atributos de la misión expresadas por los productores, se encuentra que solo toman en cuenta el elemento referido a principios organizacionales, cuando manifiestan la tradición como un valor importante en su definición de misión empresarial. No se observaron atributos relacionados con los clientes, productos o servicios, mercados, tecnología, interés por la supervivencia, crecimiento y rentabilidad.

El uso de recursos naturales en la producción agrícola implica la necesidad fundamental de cumplir con la función social de la propiedad de la tierra. En consecuencia, el compromiso con los grupos de referencia como los clientes, la competencia, el medio ambiente y la sociedad en general, es un atributo ineludible en la definición de la misión de la empresa agropecuaria, que actualmente no se encuentra presente en la misión planteada por ellos.

Por lo tanto, la misión empresarial de los productores agropecuarios, tiene como reto impostergable, el ir formulándose sobre una base clara y explicita de los propósitos de la organización, los actores y logros que se esperan alcanzar, integrando la mayor cantidad posible de los atributos antes reseñados.

Debe responder a las interrogantes, cuál es mi negocio?; pero debe ir más allá, una definición presente y añadir: cuál será el negocio?, lo que obliga al empresario a ver hacia delante y tratar de anticipar los posibles cambios en el entorno, para finalmente preguntarse cuál debería ser (Nacional financiera. Banca de Desarrollo, 2008, www.nafin.com).

Al diseñar los objetivos corporativos se proyecta la empresa hacia el futuro. La expresión "Díganos hacia donde quiere que nos dirijamos y qué resultados (rendimientos) espera de nosotros para poder diseñar y seleccionar las actividades en la forma que permita lograrlo, es una de las formas claras de expresar un concepto de objetivos, contemplados en el proceso de planificación que ocurre en toda entidad empresarial.

Una fase crítica de la planificación es la determinación de los resultados futuros, llamados objetivos, los cuales representan los resultados globales que una organización espera alcanzar en el tiempo determinado y que proporcionan las pautas hacia donde dirigir los esfuerzos y recursos (Galindo y García, 2000: 81) y el desarrollo y operacionalización concreta de su misión y visión, y los cua- 
les deben ser posibles de medir y retadores para la organización (Mariño, 2003:20). Los objetivos corporativos, ya sean a corto, mediano o largo plazo, deben ser medibles y con posibilidad de evaluación.

Según lo expresa Donnelly et al. (1994:177), los objetivos de la organización son algo más que buenas intenciones, ya que si son propuestos adecuadamente pueden:

- Convertirse en acciones específicas por realizar.

- Proporcionar dirección es decir, sirven de referencia para proponer objetivos más específicos y detallados en niveles inferiores dentro de la organización.

- Establecer prioridades a largo plazo para la organización.

- Facilitar el control de la gerencia, en virtud de constituirse en modelos de evaluación del rendimiento total de la organización.

En este sentido, Commarmond y Exiga (2001:14) resumen los objetivos en tres funciones: indicar claramente la meta, movilizar la energía hacia la meta y servir de referencia para la evaluación del resultado.

Los resultados obtenidos en sistemas de ganadería bovina en el municipio Rosario de Perijá (Tabla 3), han reseñados que los productores responsables de manejar estos sistemas consideran mayoritariamente la productividad física como aspecto sobre los cuales se formulan sus objetivos empresariales (42.55\%), seguido de objetivos planteados en función del mejoramiento de la tecnología dura de la empresa (maquinaria y equipos), con un $6.38 \%$, y por último aquellos objetivos que pretenden alcanzar, mejorar o elevar la productividad económica y gerencial (ambos con el $4.26 \%$ de frecuencia).

Los valores reseñados de aquellos productores que no establecen objetivos en su unidad productiva, alcanzaron un $42,55 \%$, lo que pudiera reflejar la no consideración anticipada de los resultados totales que se desean alcanzar como producto del esfuerzo sostenido de producción. No establecer anticipadamente el alcance de la actividad a desarrollar y los resultados y logros esperados, conlleva a

\section{Tabla 3}

\section{Distribución de los objetivos establecidos según la opinión del productor-gerente de la unidad de producción de doble propósito}

\begin{tabular}{|c|c|c|}
\hline Categoría & $N^{\circ}$ de fincas & $\%$ \\
\hline Productividad física & 20 & 42,55 \\
\hline Productividad económica & 2 & 4,26 \\
\hline Gerencial (manejo del personal, mercado, imagen, capacitación) & 2 & 4,26 \\
\hline Mejoramiento tecnología dura & 3 & 6,38 \\
\hline No respondió & 20 & 42,55 \\
\hline Total & 47 & 100 \\
\hline
\end{tabular}

Fuente: elaboración propia, 2006. 
un grado de incertidumbre en el proceso empresarial que proporciona un mayor riesgo al negocio agropecuario, a la vez que no permite canalizar sus recursos con eficacia (Stoner y Wankel, 1989).

Para que los gerentes de las empresas agropecuarias puedan organizar, dirigir y controlar todo un proceso gerencial, no cabe duda que se debe partir de un proceso de pensamiento y proyección de los diferentes escenarios que pudieran presentarse en el manejo de los recursos y la consecuente toma de decisiones acerca de cual o cuales de esas opciones son las que se llevarán a cabo para alcanzar los objetivos inicialmente planteados. Por ser procesos dinámicos, es necesario estudiar el tiempo de antelación que los productores ganaderos consideran al momento de programar y planificar sus actividades y consecuente uso de los recursos, de allí que en la Tabla 4, se encuentran señalados los referidos tiempos. Encontrándose que el mayor porcentaje lo obtuvieron aquellos productores que no realizan la planificación de sus actividades, no formulan planes, no diseñan propuestas previas de acciones por ejecutar $(23.40 \%)$, seguido de aquellos que si bien es cierto manifestaron la realización de la planificación, señalaron que no realizan esta actividad de programación de manera establecida claramente, simplemente la llevan a cabo de acuerdo a la dinámica de producción de la empresa. Un $14.89 \%$ de los productores manifestaron realizar de manera mensual labores de planificación de tareas, elaboración de planes y toma de decisiones, por lo que según lo señalado por Galindo y García (2000), se establecen solo objetivos operacionales o específicos que invariablemente son a corto plazo.

Los valores señalados en la Tabla 4, reflejan la condición particular de estos productores en cuanto a la formulación de planes de acción a corto plazo, es decir, dedicarse básicamente a la elaboración o consideración de un plan operativo, que provee respuestas específicas acerca de:

\section{Tabla 4}

\section{Distribución de respuestas acerca del tiempo de antelación considerado por los productores para planificar}

\begin{tabular}{lcr}
\hline Categoría & $N^{\circ}$ de fincas & $\%$ \\
\hline Semanal & 7 & 14.89 \\
Quincenal & 4 & 8.51 \\
Mensual & 7 & 14.89 \\
Bimensual & 2 & 4.26 \\
Anual & 1 & 2.13 \\
Otros * & 15 & 31.91 \\
No planifica & 11 & 23.40 \\
\hline
\end{tabular}

* Corresponde a productores que no indicaron tiempos precisos de planificación.

Fuente: elaboración propia, 2006. 
- El objetivo que ha de alcanzarse al finalizar el plazo de 12 meses

- Las acciones para alcanzar dicho objetivo, el momento preciso de realizarlas y tiempo de ejecución de las mismas, sobre quién recaerá la responsabilidad de la tarea asignada como meta, donde y como se llevará a cabo.

Evidentemente este tipo de planificación pareciera dejar por fuera la consideración de todos aquellos aspectos que la organización debe evaluar a los fines de concentrar los recursos en la consecución no solo de los objetivos planteados, sino de la construcción de su visión empresarial y el cumplimiento de la misión. En la planificación estratégica, se establecen los pilares de éxito en los cuales se sustentará el funcionamiento de la empresa y que corresponden a aquellas competencias o potencialidades que se poseen, así como la identificación de las competencias críticas por desarrollar 0 necesidades de mejoramiento en los que debe enfocarse para avanzar hacia el logro de su visión de futuro y poder fundamentar y sostener una ventaja competitiva a largo plazo.

El proceso de transformación de una finca en una empresa agropecuaria, se inicia primordialmente, en la sintonía que se produce al reconocer la turbulencia que representa para estas entidades productivas el verse inmersas en un escenario donde los cotidiano absorbe lo estratégico, donde la respuesta al "cómo hacerlo" es más importante a responder al "qué", donde pareciera que la clave de la subsistencia en el negocio es la velocidad de reacción, en tanto que a través de un proceso de planificación, se persigue desarrollar la "velocidad de proacción", ya que se han identificado, evaluado y seleccionado las alternativas más idóneas para la organización, enmarcadas en la naturaleza de la empresa y la proyección que esta desea poseer ante su entorno productivo más inmediato.

Por último se podría acotar que para la determinación del estado futuro deseado...... de la finca al negocio ganadero, es importante distinguir entre:

- Lo que la organización es;

- Lo que la organización va a ser;

- Lo que la organización debería ser;

- Lo que la organización puede ser

Sin embargo, la analizar los cuatro puntualizaciones anteriores, se encuentra el siguiente análisis: algunas de nuestras fincas ganaderas han decidido, tímidamente cambiar lo que eran en lo que la finca, como unidad económica y productiva, debería ser, por lo que iniciaron la evaluación de alternativas tecnológicas que le permitiera potenciar los recursos utilizados; pero igualmente encontraron que el "debería ser", algunas veces representaba un escenario demasiado utópico, y por lo tanto han decidido cambiar el "debería ser" por lo que la finca "puede ser". Si bien es cierto la individualidad de cada finca no puede cambiar el sistema productivo regional o nacional, si puede tener un impacto importante en los beneficios técnicos y económicos que como empresarios del campo pueden llegar a alcanzar aquellos productores que utilizando la planificación estratégica como herramienta de proyección del hoy en el futuro, lleguen a convertir su finca en la empresa ganadera que por largo tiempo han estado manejando. 
Esta ilustración se hace para mostrar que no sólo debe identificarse un estado futuro deseable, sino que la visión estratégica debe convertirse en mecanismos específicos para hacer de esa visión una realidad.

La planificación operativa de la empresa lleva a crear el día a día de la organización. Cada una de las distintas unidades constituyentes de la organización, de negocios y funcionales, necesita desarrollar planes detallados en términos operativos o tácticos con base a un plan general de la compañía (Goodstein et al., 2004).

Los planes operativos o tácticos, según Schermenrhorn (2002:141), definen lo que necesita hacerse en áreas específicas para implementar planes estratégicos y alcanzar metas también estratégicas. Entre ellos se tienen los planes de producción, los planes financieros, relacionados con el dinero requerido para apoyar las diversas operaciones, los planes de instalaciones, los planes de mercadotecnia, que se refiere a los requerimientos de la venta y distribución de bienes y servicios; y los planes de recursos humanos.

Al ser los planes el resultado del proceso de planificación que consiste en el diseño de esquemas detallados de lo que habrá de hacerse en el futuro y las especificaciones necesarias para realizarlos, debe conocerse que en el seno de toda empresa, los planes se disponen en diferentes niveles de la organización, en relación con la estructura organizativa de la misma. Tal como lo señala Stoner y Wankel (1989:103), en cada nivel, los planes cumplen con dos funciones efectivas en relación con:
1. Indican los objetivos a alcanzar por los planes diseñados

2. Identifican, cuantifican y ordenan los medios, recursos y técnicas para alcanzar los objetivos incluidos en el plan estratégico de la empresa.

Pero de igual forma, los planes elaborados a mediano no son suficientes para lograr que las operaciones, cotidianas se realicen con los detalles y cronogramas que conllevan.

La diversidad de recursos que intervienen en el proceso productivo ganadero, se ha utilizado y adaptado el gráfico propuesto por Donnelly et al. (2001) dividiendo los planes en:

- Planes de manejo: los cuales contemplan las actividades correspondientes al manejo del recurso rebaño (tipo de suplementos considerados a la hora de planificar, criterios considerados en la actividad reproductiva, planificación en el manejo del rebaño, plan sanitario) e infraestructura (criterios utilizados en la planificación del mantenimiento de maquinarias y equipos, mantenimiento de construcciones) utilizados en la actividad productiva.

- Planes de recurso humano: se refiere básicamente a los criterios considerados en la planificación del personal, en cuanto a la selección del personal a contratar en la empresa ganadera.

- Plan administrativo: contempla algunos elementos considerados al momento de planificar los aspectos de carácter administrativo, tales como: aspectos administrativos tomados en cuenta al momento de planificar y fuentes de información. 
Los planes de manejo estudiados y referidos al recurso rebaño, comprenden aspectos de alimentación, sanidad y reproducción. Normalmente, se incluyen planes de producción, que se refieren a los métodos y la tecnología que necesita la gente en el trabajo (Schermenrhorn, 2002). En la Tabla 5, se encuentran señalados los resultados obtenidos en las respuestas recibidas de los productores en relación con los tipos de suplementos considerados a la hora de planificar. Allí se observó que la mayor proporción la presentan los suplementos sal, minerales, melaza y alimento concentrado $(100,93.62,89.36$ y $57.45 \%$, respectivamente). En investigaciones posteriores se está evaluando esta planificación con la aplicación real de lo planificado, de manera tal que se pueda medir la conveniencia y efectividad del proceso de planificación llevada a cabo por los productores.

Las decisiones que se toman referidas al manejo operativo de las activida- des reproductivas del recurso rebaño (Tabla 6) son efectuadas según el tipo de monta $(23.40 \%)$, la época del año $(14.89 \%)$, la sincronización del celo $(46.81 \%)$, el diagnóstico de preñez $(97.87 \%)$ y condición fisiológica del animal (89.36\%). La práctica cotidiana de los productores contempla la consideración de alguno de estos criterios de manera individual o la combinación entre ellos.

Siendo los animales que componen el rebaño, un recurso considerado por el productor ganadero como muy sensible e importante dentro de su sistema productivo, la atención prestada por ese productor a todas las actividades que tienen relación con el mismo, se refleja evidente en los resultados señalados en la Tabla 7, los cuales indican valores que se encuentran, en su mayoría, por encima del $50 \%$ de frecuencia para la planificación de las actividades de separación de animales por lote $(95.74 \%)$, herraje e identificación y descorne $(97.87 \%)$, castración $(59.57 \%)$, pesaje de animales

Tabla 5

Distribución de respuestas acerca del tipo de suplementos alimenticios considerados a la hora de planificar

\begin{tabular}{lcc}
\hline \multicolumn{1}{c}{ Categoría } & $N^{\circ}$ de fincas & $\%$ \\
\hline Alimento concentrado & 27 & 57.45 \\
Sal & 47 & 100.00 \\
Minerales & 44 & 93.62 \\
Melaza & 42 & 89.36 \\
Bloques nutricionales & 1 & 2.13 \\
Heno & 4 & 8.51 \\
Silaje & 3 & 6.38 \\
Cebada & 18 & 38.30 \\
Yacija & 9 & 19.15 \\
\hline
\end{tabular}

Fuente: elaboración propia, 2006. 


\section{Tabla 6}

\section{Distribución de los criterios considerados en las actividades reproductivas}

\begin{tabular}{lcc}
\hline \multicolumn{1}{c}{ Categoría } & $N^{\circ}$ de fincas & $\%$ \\
\hline Tipo de monta & 11 & 23.40 \\
Epoca del año & 7 & 14.89 \\
Sincronización del celo & 22 & 46.81 \\
Diagnóstico de preñez & 46 & 97.87 \\
Condición fisiológica del animal & 42 & 89.36 \\
\hline
\end{tabular}

Fuente: elaboración propia, 2006.

Tabla 7

\section{La planificación del manejo integral del rebaño}

\begin{tabular}{lcc}
\hline \multicolumn{1}{c}{ Categoría } & $N^{\circ}$ de fincas & $\%$ \\
\hline Tatuaje & 22 & 46.81 \\
Herraje e identificación & 46 & 97.87 \\
Descorne & 46 & 97.87 \\
Castración & 28 & 59.57 \\
Pesaje de animales & 35 & 74.47 \\
Pesaje de la leche & 32 & 68.09 \\
Baños para el ganado & 36 & 76.60 \\
Separación de animales por lote & 45 & 95.74 \\
\hline
\end{tabular}

Fuente: elaboración propia, 2006.

$(74,47 \%)$, pesaje de la leche $(68.09 \%)$, baños para el ganado $(76.60 \%)$ y tatuaje $(46.81 \%)$.

De igual manera, la sanidad animal es un aspecto de manejo animal considerado en el $100 \%$ de las unidades de producción estudiadas, tanto en lo referido a las vacunaciones, desparasitación y suministros de vitaminas.

Los planes de manejo de la infraestructura física comprendida por el mantenimiento de maquinarias y equipos, instalaciones y construcciones, son los que tienen que ver con las instalacio- nes y diseños de trabajos requeridos para apoyar las actividades (Schermenrhorn, 2002), representan una actividad tradicionalmente considerada por el productor ganadero dentro del manejo de negocio que lleva en su finca, en virtud de la inversión monetaria que representan estos elementos dentro de la totalidad de capital invertido. Sin embargo, los resultados obtenidos señalan que solo el $31.91 \%$ de los productores entrevistados manifiestan hacer una planificación de las actividades de mantenimiento de maquinarias y equipos (Gráfico 1). 
En tanto que, solo el $25.53 \%$ de los productores realizan la planificación de mantenimiento e instalaciones productivas (Gráfico 2). Esto pudiera explicarse por la presión monetaria que expresan sentir los productores al tener que distribuir el escaso dinero entre todos los requerimientos propios de la actividad productiva.

El proceso de decidir consiste en que, existiendo varias posibilidades, que se expresan en un conjunto de alternativas, se elige una de ellas para realizar la acción, o bien para no realizarlas. Por supuesto que en este proceso de decisión considera importante el considerar la mayor cantidad de información sobre datos físicos y económicos que existan a través de las diferentes fuentes de donde se obtiene la misma, así como las situaciones favorables o no que ocurren en el entorno. Consultas a profesionales, visitas a otras unidades de producción, suscripción a revistas especializadas, consulta con instituciones oficiales del sector, registros de producción y resultados obtenidos en la misma empresa constituyen alguna de las fuentes posibles de consulta ante de elaborar un plan o tomar una decisión.

Al estudiar los planes de carácter administrativo (Gráfico 3), se pudo observar que los aspectos considerados corresponden a recursos con que cuenta $(65.96 \%)$, precios de los productos $(55.32 \%)$, costos de los insumos $(40.43 \%)$, según las necesidades presentadas y prioridades identificadas $(6.38 \%)$.

Las decisiones a ser tomadas en estas organizaciones (Gráfico 4), están

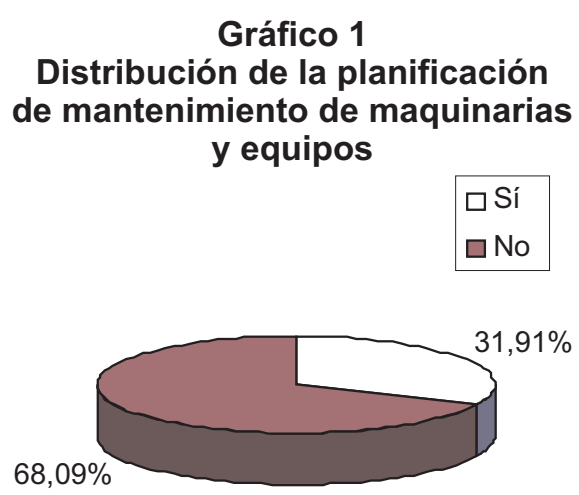

Fuente: elaboración propia, 2006.

Gráfico 2

Planificación de mantenimiento de construcciones e instalaciones

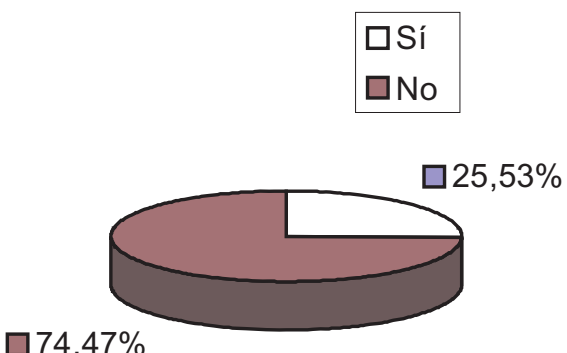

Fuente: elaboración propia, 2006.

basadas en el fuente y tipos de información que maneja el productor, tal es el caso de publicaciones escritas (19.15\%), asociación y amigos (74.34\%). Esto discrepa de lo señalado por (Margüendi, 1987).en cuanto a considerar que con el empleo de la información procedente de la propia empresa se puede analizar los resultados compararlos con la planificación realizada y los resultados de otras empresas similares.

Los planes de manejo para el recurso humano, se pueden definir (Infan- 


\section{Gráfico 3}

\section{Los aspectos administrativos que toman en cuenta que toma en cuenta para planificar}

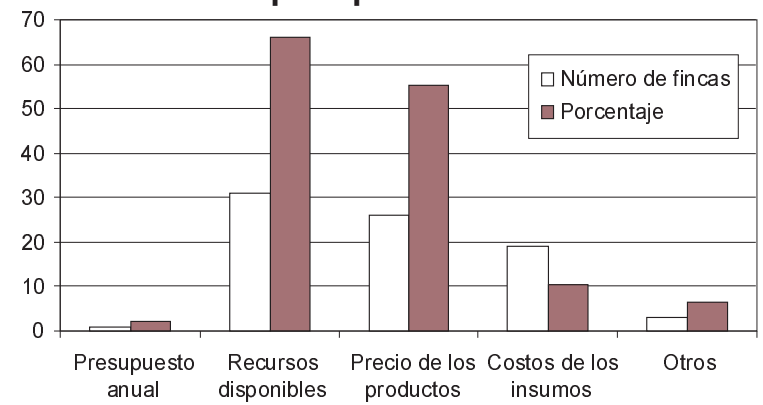

*Según las necesidades presentadas, necesidades más urgentes, prioridad de necesidades. Fuente: elaboración propia, 2006

Gráfico 4

Fuentes y tipos de información utilizadas para tomar decisiones

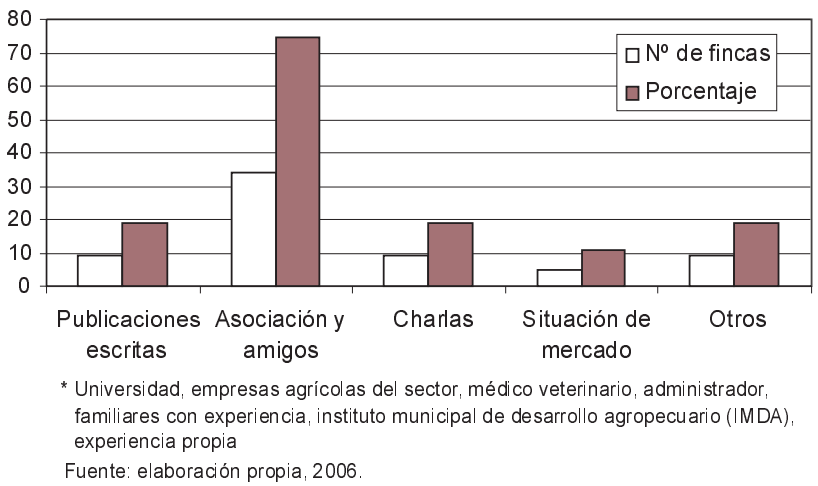

te, 2003:23) como el proceso de anticipar y prevenir el movimiento de personas hacia el interior de la organización, dentro de ésta y hacia fuera. Según Schermenrhorn (2002:141), los planes de recursos humanos, se enfocan hacia el reclutamiento, selección y colocación de personas en diferentes puestos de trabajo.

Su propósito es utilizar estos recursos con tanta eficacia como sea posible, donde y cuando se necesiten, a fin de alcanzar las metas de la organización. Pero evidentemente, este proceso requiere a su vez de un proceso de escogencia del individuo adecuado para el cargo adecuado, por lo que contempla un proceso de comparación seguido de un proceso de decisión. La situación de planificación del recurso humano en los sistemas de producción ganaderos bajo observación, estuvo básicamente estudiado en relación a los criterios utilizados para seleccionar el personal que labora en las unidades de producción, puesto que aspec- 
tos tales como contratación del personal, programas de capacitación e incentivos, generalmente se realizan, en el caso de que se hagan, sin ningún tipo de programación previa, obviando de esta manera cualquier proceso de planificación.

Al momento de seleccionar el personal que formará parte del recurso humano de la finca, un $53.19 \%$ de los productores utilizan la experiencia del trabajo como criterio de mayor peso para decidir sobre su contratación o no (Tabla 8); aun cuando esa experiencia referida por el propio interesado en conseguir el trabajo, pocas veces es verificada previamente por el contratante. La disponibilidad inmediata que posea el individuo que solicita el puesto de trabajo es el segundo criterio más utilizado $(51.06 \%)$ en la selección del personal, lo que pudiera reflejar que realmente no se trata de un criterio consistente con la obtención de un personal idóneo para ocupar el cargo disponible, puesto que solo un $8.51 \%$ de los productores reconocen o utilizan el nivel de capacitación de ese solicitante como criterio que permita realizar el proceso de selección. En este sentido, Infante
(2003:37) expone que es necesario planificar la fase de reclutamiento partiendo de que no se puede dejar a un lado la descripción del cargo para el cual se requiere personal.

La modalidad de establecer un período de prueba como mecanismo que permita evaluar las habilidades, destrezas y conocimientos que posea el individuo solicitante en cuanto al cargo propuesto para ocupar, no fue en ningún caso considerado por estos productores, aun cuando como lo señala Infante (2003), esta modalidad pudiera ofrecer como ventaja a la empresa, la seguridad de contratar la persona adecuada para el cargo y como desventaja el peso financiero de descartar ese individuo y tener que iniciar nuevamente el proceso de selección.

\section{Conclusiones}

La evidencia empírica analizada en esta investigación muestra que la planificación estratégica agropecuaria llevada a cabo por los productores esta básicamente caracterizada ejecutar una planifi-

\section{Tabla 8}

\section{Criterios para seleccionar el personal}

\begin{tabular}{lcc}
\hline \multicolumn{1}{c}{ Categoría } & $N^{0}$ de fincas & $\%$ \\
\hline Experiencia & 25 & 53.19 \\
Documentación de identificación & 7 & 14.89 \\
Recomendaciones o referencias & 17 & 36.17 \\
Disponibilidad inmediata & 24 & 51.06 \\
Nivel de capacitación & 4 & 8.51 \\
Otros * & 3 & 6.38 \\
\hline
\end{tabular}

*Amistad, necesidad de personal, comportamiento, con ganas de trabajar.

Fuente: elaboración propia, 2006. 
cación del día a día, obviando los planes y proyecciones a largo plazo, y ocupándose principalmente del recurso animal, que sin dejar de ser importante, no constituye la totalidad del sistema productivo.

Al contrastar la vivencia de planificación presente en estas empresas con el referencial teórico que existe, se puede concluir que los responsables de estas empresas desarrollan un proceso de planificación estratégica que se ejecuta en función de una apreciación de cómo se desea ser percibido como empresario, con una misión empresarial intrínseca referida a valores de tradición familiar y con planes operativos puntuales sin conexión de interdisciplinaridad, lo que evidentemente dista de los criterios reseñados para la construcción y definición de la misión, visión, objetivos y planes empresarial.

Es necesario que la planificación estratégica agropecuaria sea considerada a la luz de la características propias del negocio agrícola, entre ellas, la incertidumbre, la inseguridad en el medio rural, la variabilidad de precios de insumos y productos agrícolas que posiblemente determinen un nuevo paradigma para la gerencia en este medio, dado que el referencial teórico manejado por los especialistas de la gerencia moderna, dista en la mayoría de sus aspectos de lo caracterizado en los sistemas agropecuarios estudiados y sin embargo sigue existiendo evidencia de la permanencia de estos empresarios en el negocio agrícola con rendimientos económicos y físicos, por lo menos satisfactorio para ellos.

La planificación estratégica agropecuaria que destaca en estas organizaciones es un proceso complejo de toma de decisiones llevada a cabo de manera dinámica y flexible que considera la racionalidad económica como principal componente en el diseño de objetivos y planes operativos, y otorga atención a situaciones inmediatas más que aquellas a largo plazo, por lo que se convierte principalmente en una planificación de carácter táctico más que estratégica.

\section{Referencias bibliográficas}

Acosta Fabián y Calvi Mariana (2008). Gestión de la empresa ganadera. Versión digitalizada. Htpp: www.produccionbovina.com/empresa_agropecuaria/empresa_agropecuaria. Revisado el 23 de Abril de 2008

Commarmond, Gisele y Exiga Alain (2001). Cómo fijar objetivos y evaluar resultados. Colección Gerencia Empresarial. El Nacional. Venezuela. $166 \mathrm{pp}$

David Fred (1997). Conceptos de Administración Estratégica. Quinta Edición. Prentice-Hall Hispanoamericana S.A. 355 pp.

Donnelly James, Gibson James, Ivancevich John (1994). Dirección y administración de empresas. Addison-Wesley Iberoamericana. $848 \mathrm{pp}$.

García Anton. y Acero de la Cruz Raquel (2008). Gestión de empresas ganaderas y veterinarias. Versión digitalizada. http://www.uco.es/dptos/prodanimal/economia/APOYODOC/libro \%20gestion/capi1 Revisado 23 Abril 2008.

González Baldomero (1992). Ganadería mestiza a base de pastos en condiciones húmedas y subhúmedas de la Cuenca del Lago de Maracaibo. En: González-Stagnaro (Ed) Ganadería Mestiza de Doble Propósito. Maracaibo, Venezuela. Ediciones Astro Data. 367-379 pp. 
Goodstein Leonard, Nolan Thimothy, Pfeiffer William (2004). Planeación para planear. Planeación Estratégica Aplicada. Mc-Graw-Hill Interamericana. Colombia. 442 pp.

Griffin Ricky y Ebert Ronald (1997). Negocios. México. Prentice-Hall Hispanoamericana, S.A. Cuarta edición. 761 pp.

Hernández y Rodríguez Sergio (2006). Introducción a la administración. Teoría general administrativa: origen, evolución y vanguardia. Planeación estratégica. México. Mc Graw-Hill Interamericana Editores. 311-312.

Hernández Roberto, Fernández-Collado Carlos, Baptista Pilar (1991). Definición del tipo de investigación a realizar: básicamente exploratoria, descriptiva, correlacional o explicativa. Metodología de la investigación. México. Mc Graw-Hill Interamericana Editores. 57-73 pp.

Hill Charles y Jones Gareth (2001). Administración estratégica. Un enfoque integrado. McGraw Hill. Tercera edición. Colombia. 540 p.

Hurtado Jacqueline (1998). Metodología de la investigación Holística. Sypal. Caracas. $594 \mathrm{p}$.

Hurtado Jacqueline (2002). El como o la metodología de la investigación. El proyecto de investigación holística. Cooperativa Editorial Magisterio. Bogotá. $135 \mathrm{p}$.

Infante Cora (2003). Manual de Gestión de Recurso Humano. Universidad Nacional Experimental del Táchira (UNET).

Marino M. Empresa Agropecuaria. Introducción a las Ciencias Agrarias. Facultad de Ciencias Agrarias. UNMDP. 2009. www.mdp.edu.ar/agrarias/grado/100/ archivos/Empresas_Agropecuarias_ 2007.doc
Mariño Hernando (2003). Gerencia de procesos. Alfaomega colombiana S.A. 147 p.

Marguiegui Antonio (1987). Administración de empresas agropecuarias. Fundación La Salle de Ciencias Naturales. Colección de cuadernos FLASA. Serie Ciencia y Tecnología N. 1. 215 p.

Münch Galindo Lourdes (2000). Fundamentos de administración. $5^{\text {ta }}$ Edición. México. $240 \mathrm{p}$.

Nacional financiera. Banca de Desarrollo, 2008, www.nafin.com. Revisada el 23 de abril del 2008.

Peña María, Urdaneta Fátima, Arteaga Gustavo y Casanova Angel. (1999). Características personales y actitudinales del productor gerente de empresas de ganadería bovina de doble propósito en los municipios Rosario y Machiques de Perijá. Revista de la Facultad de Agronomía. 16 supl. 1:259264.

Olivera Carlos (2009). La misión: ¿Slogan o expresión concentrada de la estrategia organizacional? Herramientas para emprendedores._http://www. gestiopolis. com/innovacion-emprendimiento/mision-slogan-o-expresionconcentrada-de-la-estrategia-organiz acional.htm

Schermerhorn John (2002). Planeación: El ingrediente esencial para trazar el rumbo. Administración. Editorial Limusa. México. Primera Edición. 524 pp.

Serna Gómez Humberto (1997). Gerencia estratégica. Planeación y gestión. Teoría y metodología. Global Ediciones. $385 \mathrm{p}$.

Storner James y Wankel Charles (1989). Administración. Tercera Edición. Prentice-Hall Hispanoamericana. 826 pp. 